تحليل عملي لاراسة تأثير العمق ونوع التربة على الاجهادات المتولاة على ساق المحراث اثناء عملية الحراثة الاجنة

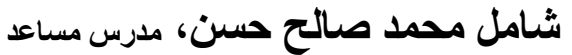

كلية الزراعة والغابات / جامعة الموصل

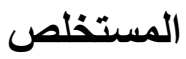

اجريت الدراسة لغزض تحليل الاجهادات المتولدة على سـاق المحراث عند اختلاف العمق ونـوع التربـة، وقابلية تحمله

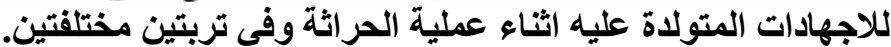

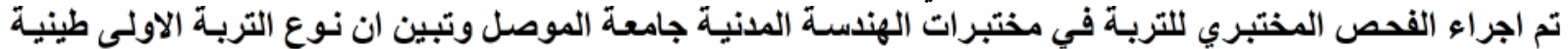

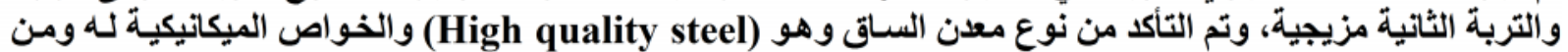

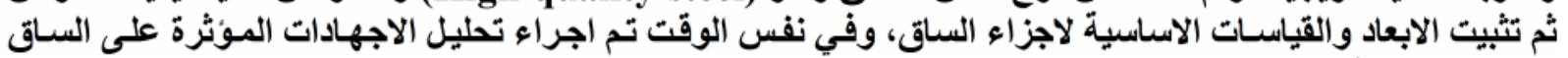

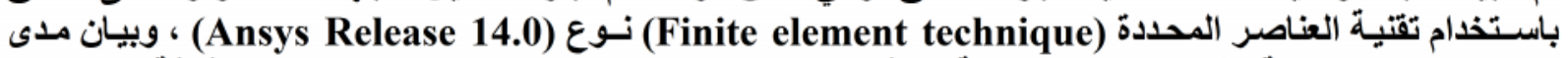

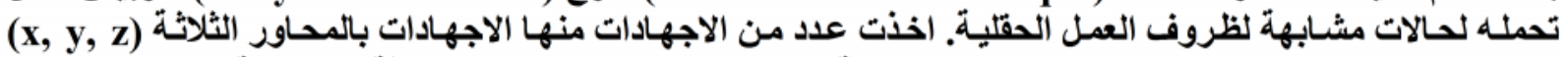

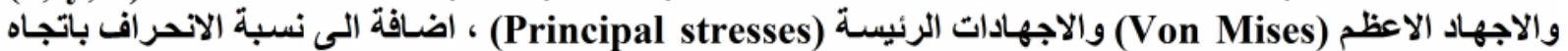
.$(\mathbf{x}, \mathbf{y}, \mathbf{z})$

مرحلة العملية تثبيت عدد من المجسـات (strain gauges) على سـات المحراث وقياس الأ تب، حيث

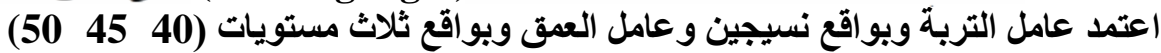

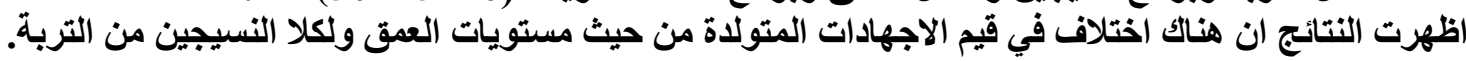

\title{
Practical Analysis for Studying the Effect of Both Depth and Soil types on the Stresses Generated on the draw-bar During Plowing Process
}

\author{
Shamil M. Hassan, Assistant lecturer \\ Coll. of Agric. Univ. of Mosul
}

\begin{abstract}
A study was conducted for the purpose of determining the stresses generated on the draw-bar as a result of different types of soil and depth produced during plowing process in two different soils.

Laboratory analysis was conducted for studying the soil analysis in the department of Civil Engineering University of Mosul.

Analysis revealed that there were two different types of soil (clay and mixed). At the same time mechanical properties and basic measurements of draw-bar was carried out. At the same time stress analysis of draw-bar was done using finite elements program (Ansys releases 14.0 types), to determine the properties during application in different stress analysis parameters where used $(x, y, z)$ and Von

Mises in addition to principal stresses and percentage deviation of the direction of the three axes $(x, y, z)$.

During practical phrase, installation of strain gauges on draw-bar was done, for two types of soil at different depth level factors using three level $(40,45,50) \mathrm{cm}$.

The present results revealed that there was a difference in the stress values generated in relation to depth and types of soil.
\end{abstract}

Keywords : Soil Test, Stress Analysis, Finite Element Analysis, Draw-bar 
:

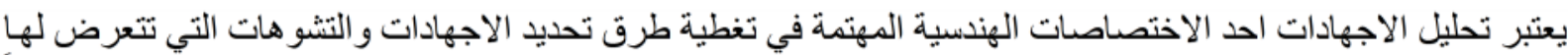

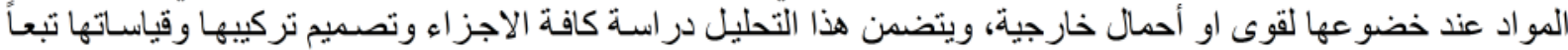

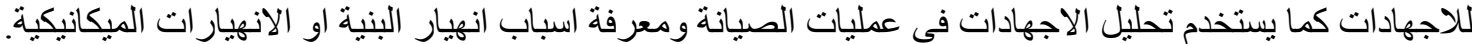

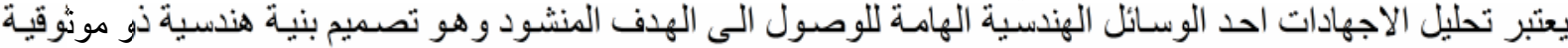

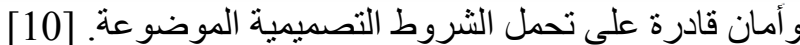

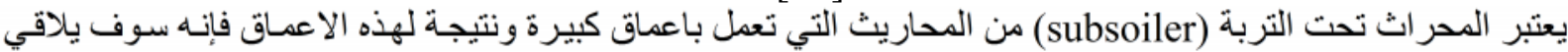

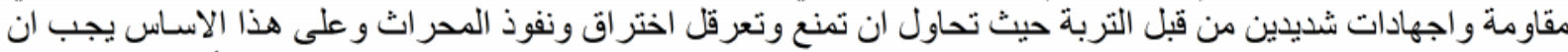

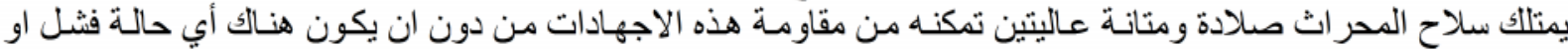

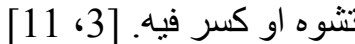

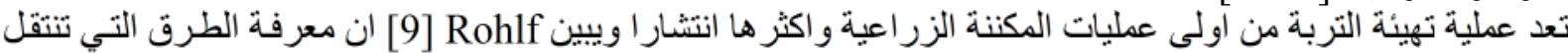

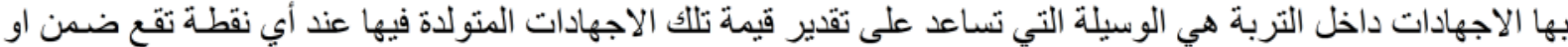

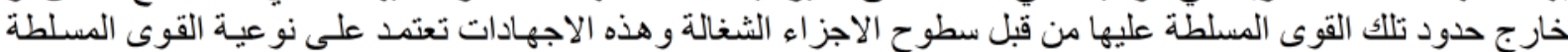

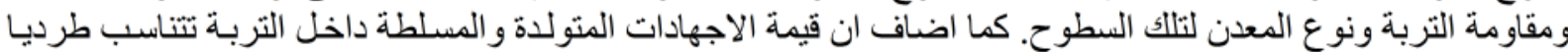

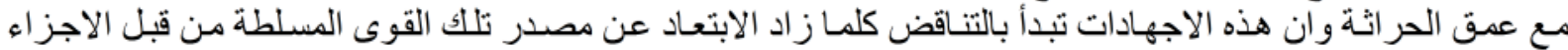
الثغالة.

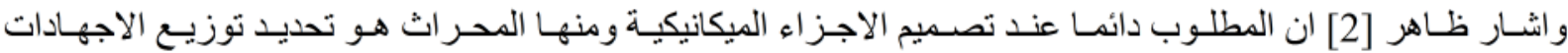

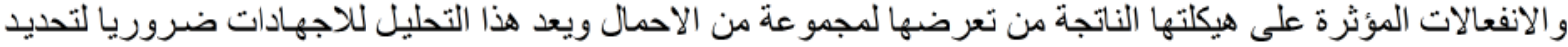

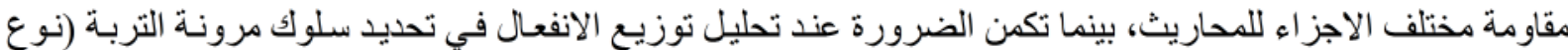

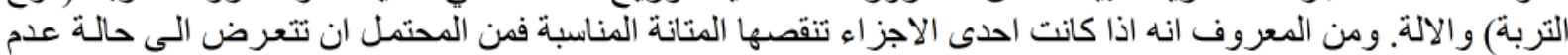

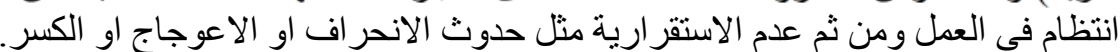

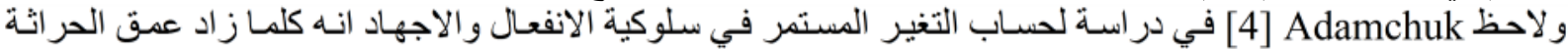

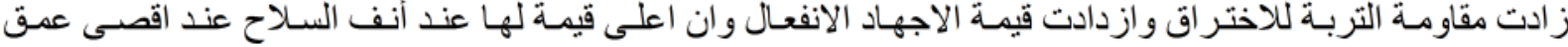
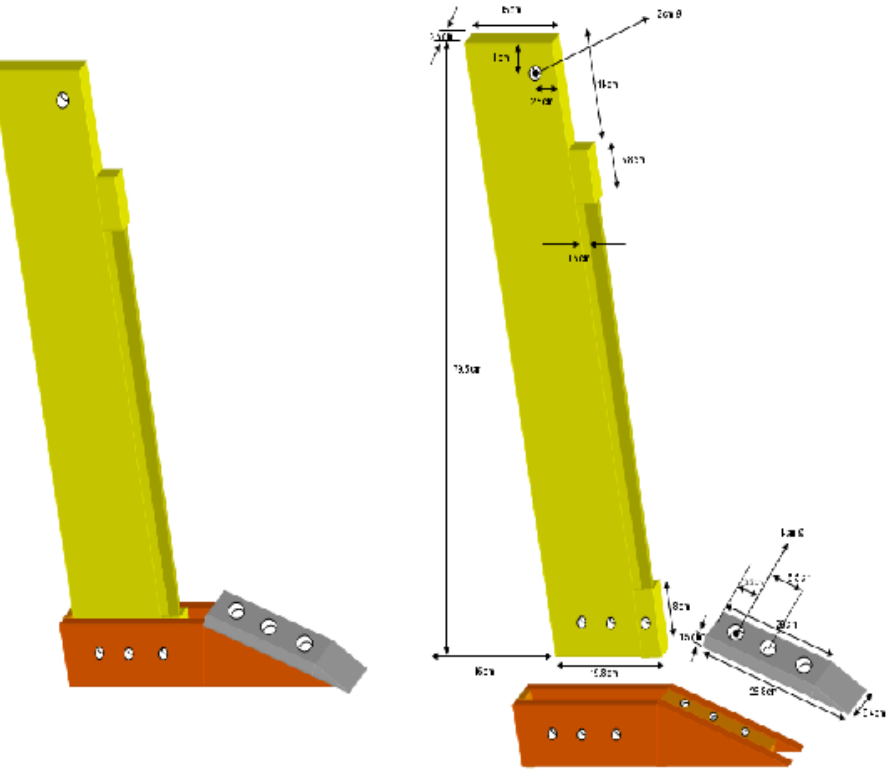

(1)
مواد البحث وطر ائقهه :

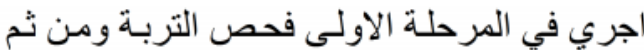

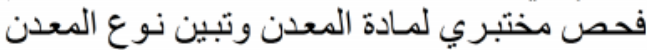

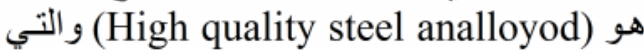

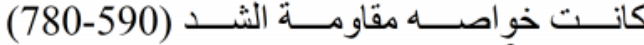

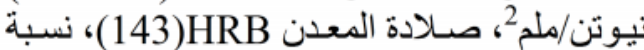

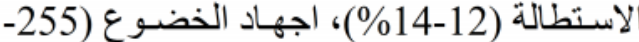

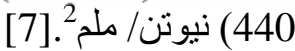

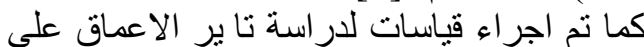

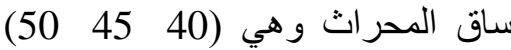

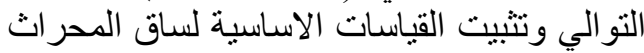

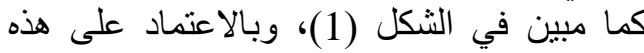
القياسات والابعاد تم توزيع الاجهادات الاتئ المؤثرة

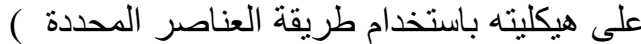
( Ansys Finite element ) Release 14.0) له لـ

الاجهادات من خلال النتائج التي تحمل الحصـول عليها نتيجة القوى المسلطة عليه و التي يتم حسابها باستخدام المعادلة (1). [5]

$\mathrm{D}-\mathrm{Fi}\left[\mathrm{A}+\mathrm{B}_{(\mathrm{s})}+\mathrm{C}_{(\mathrm{s})}{ }^{2}\right] . \mathrm{WT}$ 
حيث ان : موة سحب الالة (نيوتن) : معان

: معامل بعتمد على نسجة التربة : F

: A, B, C

: سر عة الحر اثة (كيلومنز / ساعة) : S

i

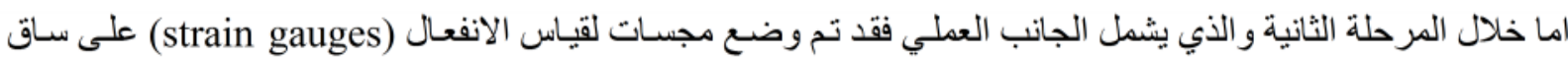

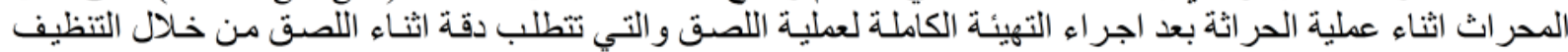

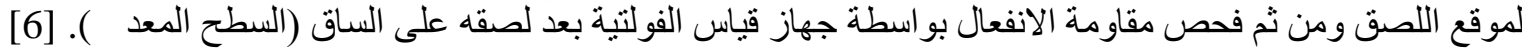

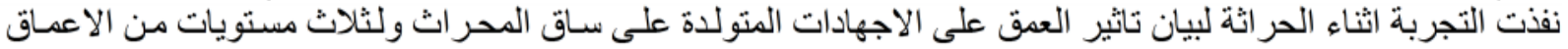

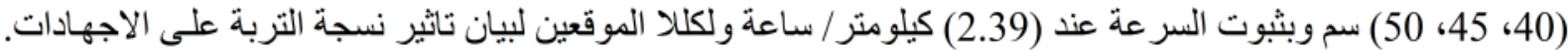
ويبين الجدول (1) مكونات نسيجة التربتين .

(1) (1) مكونات نسجة التربتين

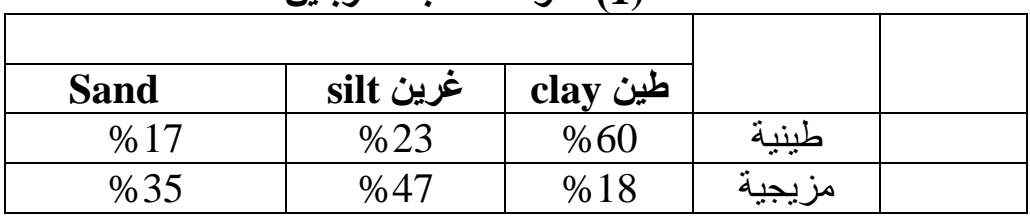

نلاحظ من الاشكال الخاصة بتوزيع الاجهادات على بدن الساق المحر اث تحت التربـة ان النتائج التـي تم الحصــول عليها

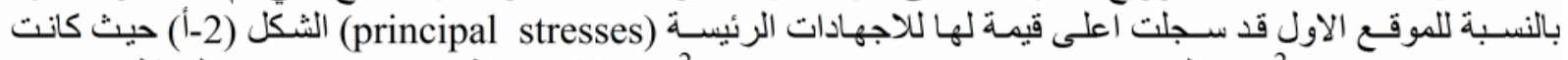

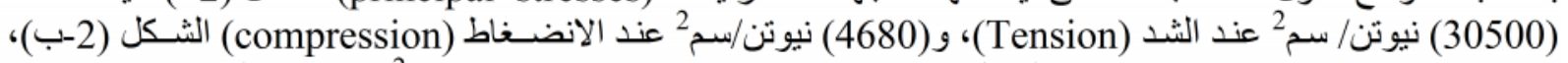

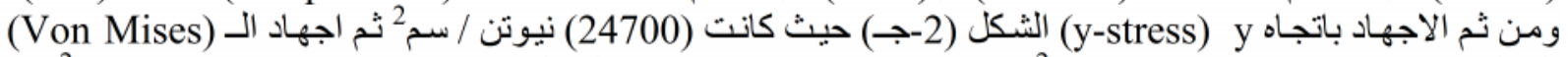

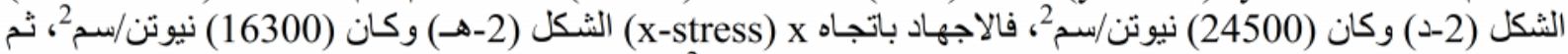

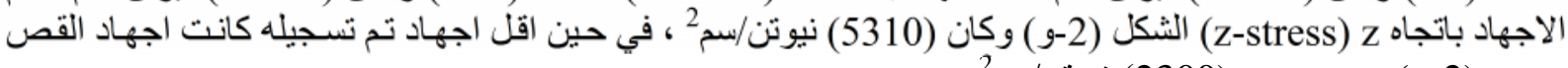
ن 2300) نيوتن/سم (2)

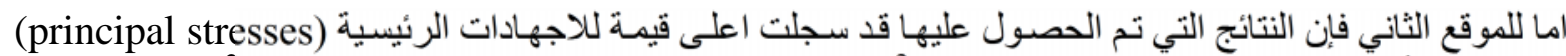

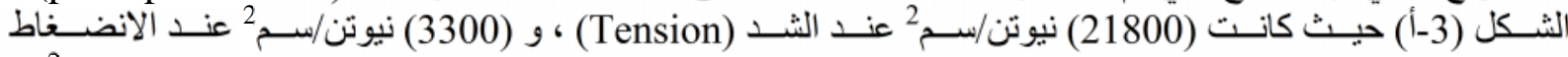

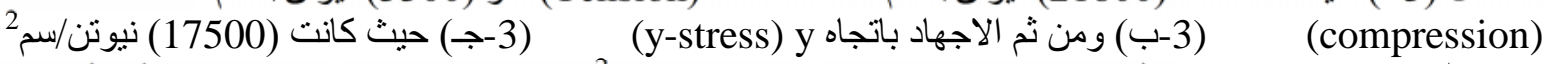

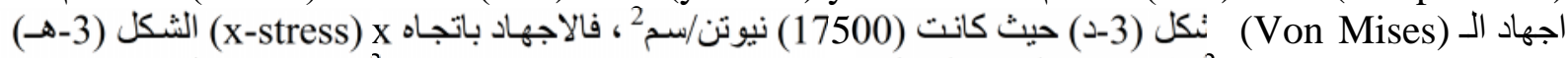

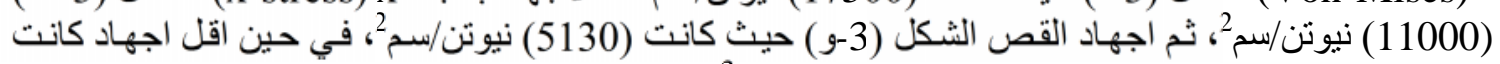

$$
\text { (3750) (-3) ( }
$$

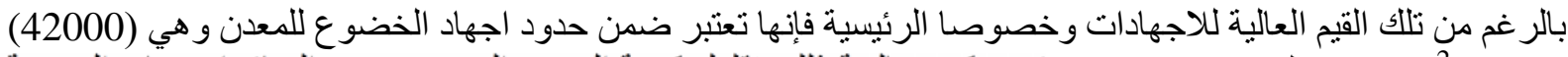

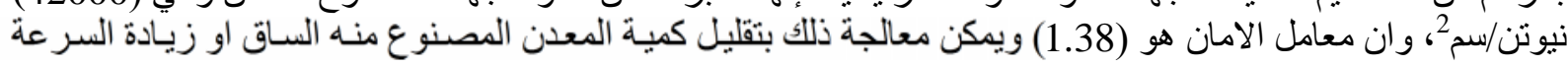




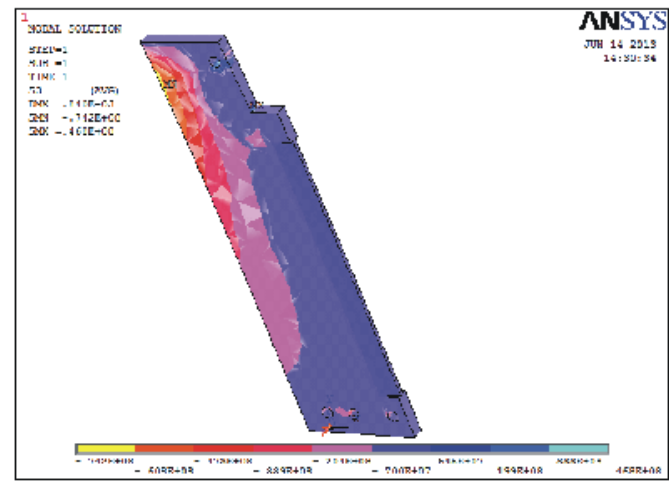

Compression ( -2)

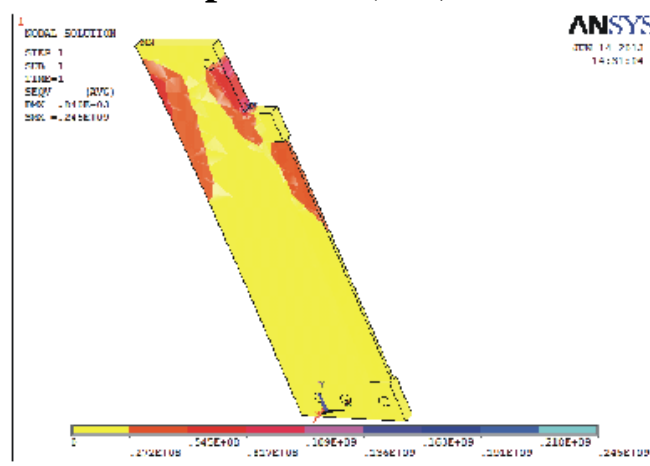

Von mises ( -2)

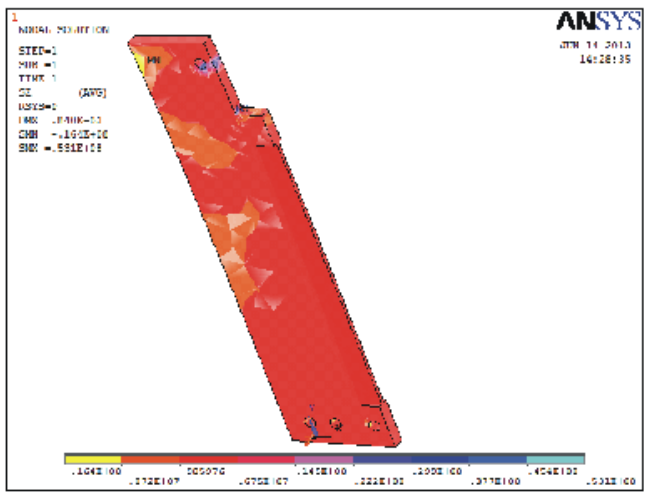

Z-stress ( -2)

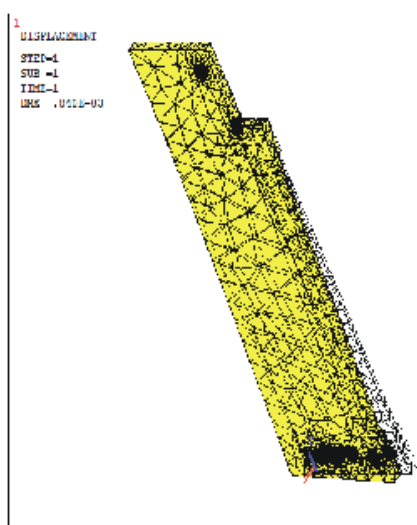

( -2)

deformed and undeformed shape

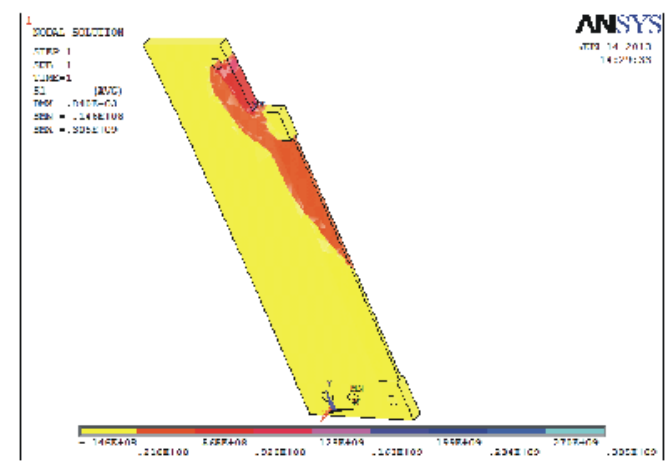

Tension ( -2)
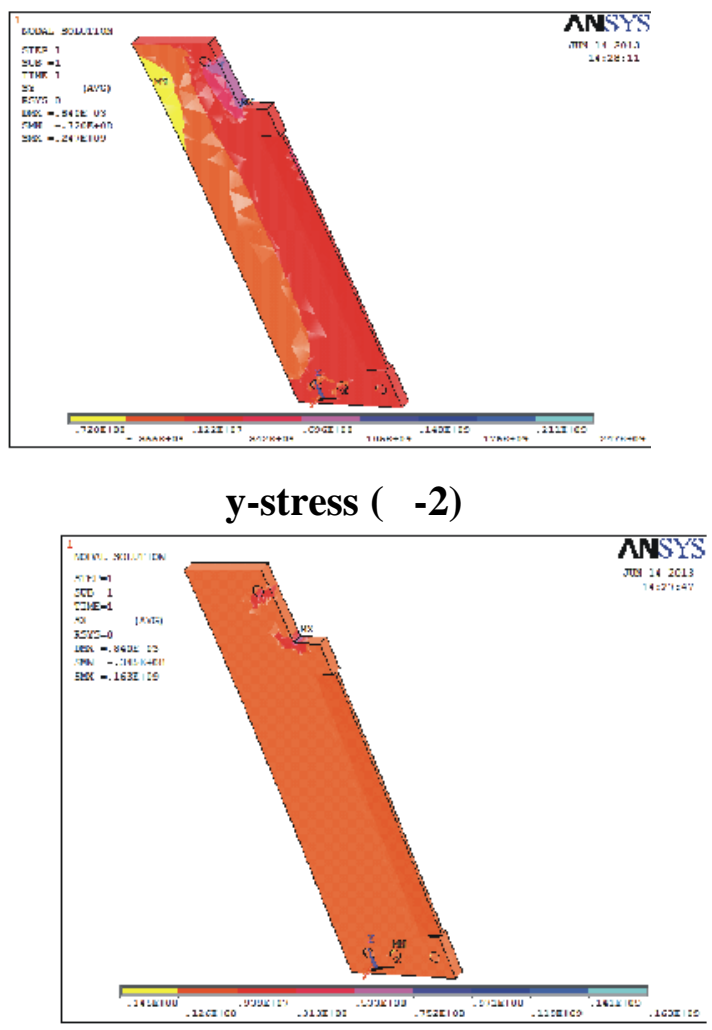

X-stress (-2)

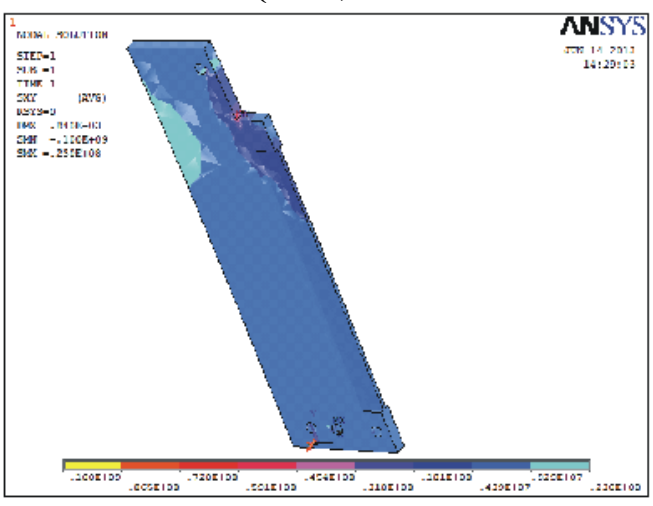

Shear xy ( -2) 
: تحليل عملي لاراسة تأثير العمق ونوع التربة على الاجهادات المتولدة على ساث المحراث اثناء عملية الحراثة

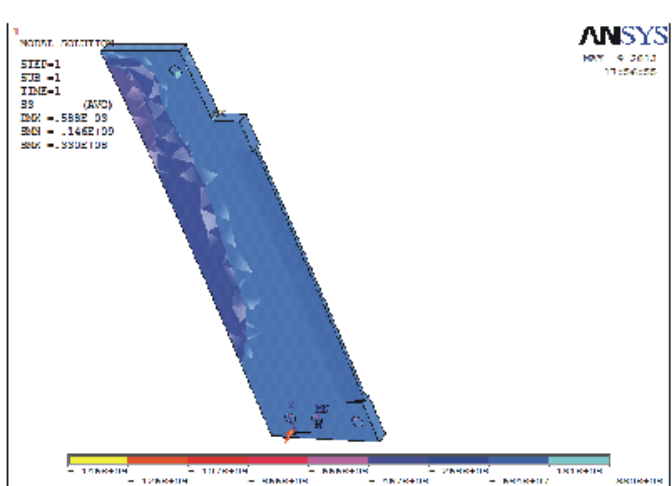

Compression ( -3)

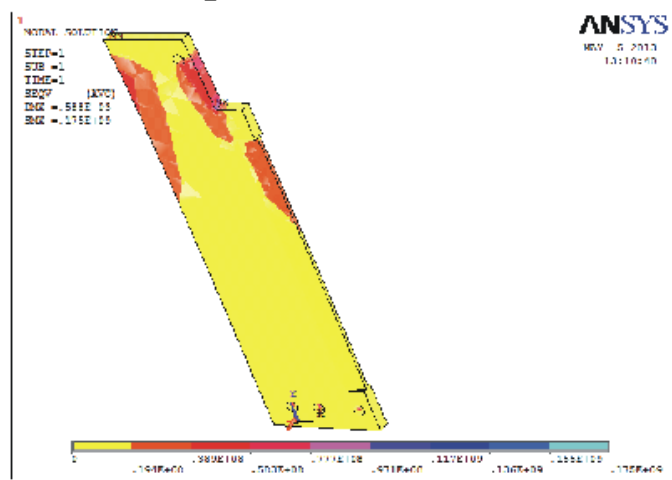

Von mises ( -3)

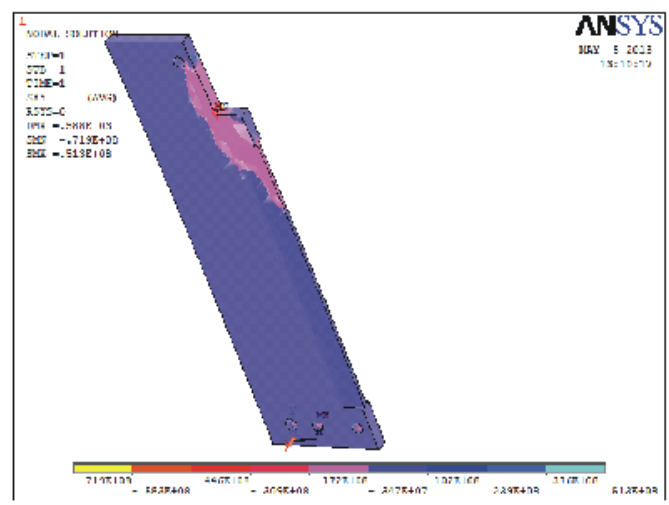

Shear xy $(-3)$

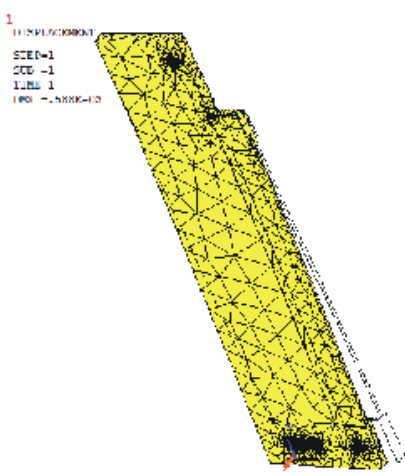

$(-3)$

deformed and undeformed shape

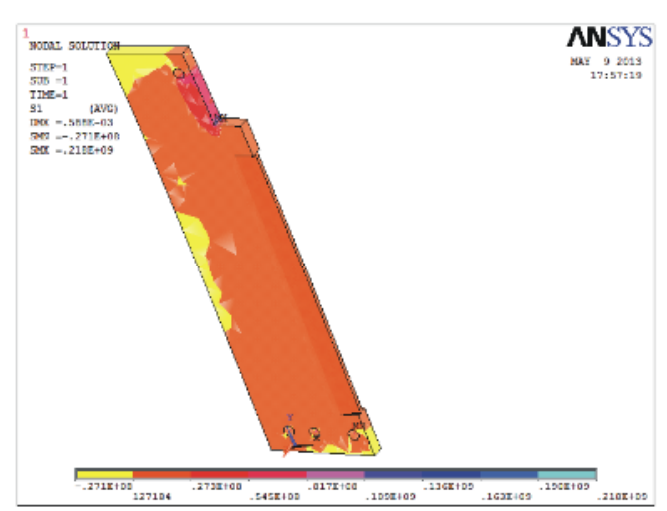

Tension ( -3)
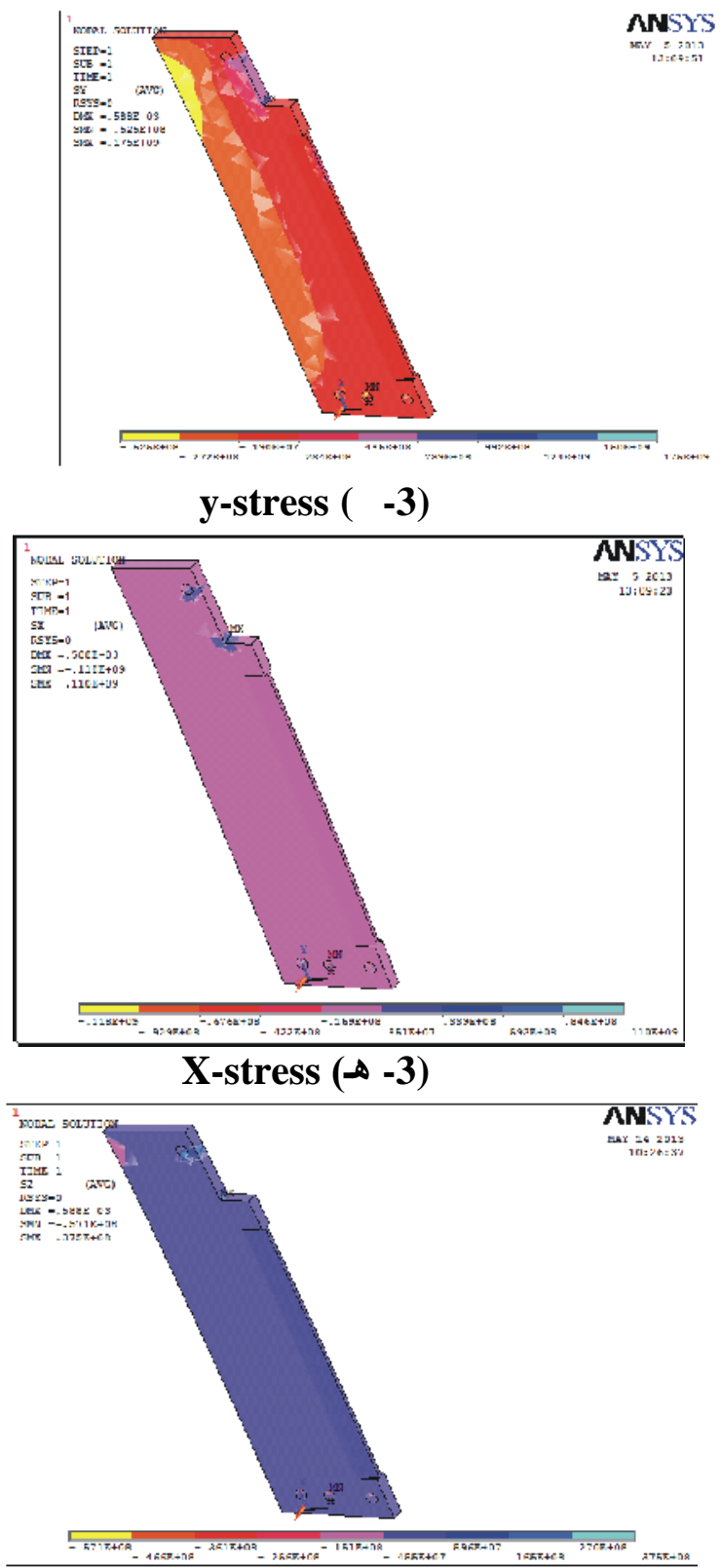

Z-stress ( -3) 


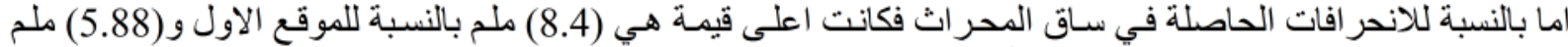

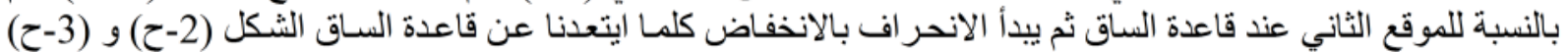

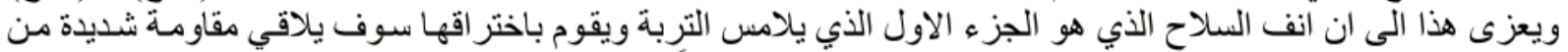

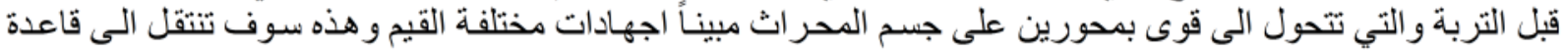

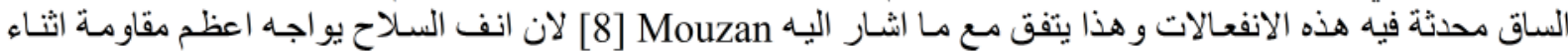

ويوضح الجدول (2) قيم الاجهادات على سـاق المحر اث لموقعين مختلفين و لاعماق مختلفة و عند سر عة 2.39 كيلومتر /

(2) قيم الاجهادات لموق ين مختلفين

\begin{tabular}{|l|c|c|c|c|c|c|c|}
\hline \multicolumn{2}{|c|}{$($ الاجهادات (نيوتن/سم² } & \multicolumn{3}{|c|}{ ( ) } & ( / ) & \\
\hline 29280 & 25700 & 22800 & 50 & 45 & 40 & 2.39 & \\
\hline 21360 & 20850 & 20490 & 50 & 45 & 40 & 2.39 & \\
\hline
\end{tabular}

يلاحظ من قيم الاجهادات التي تم الحصول عليها و عند نفس العمق للموقعين ان قيم الاجهادات في الموقع الاول اعلى مما

(1)

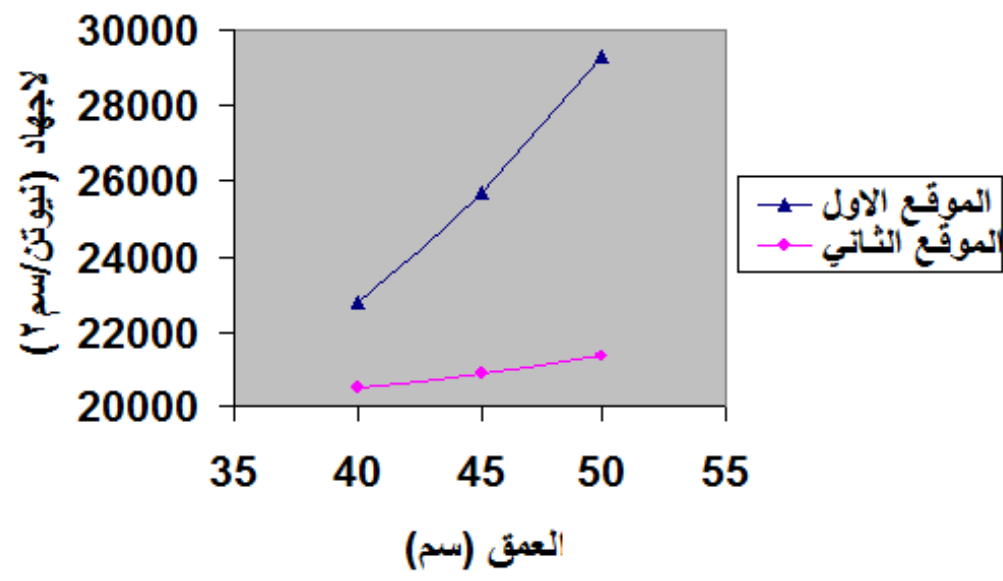

- (4) مخطط اجهاد - (4)

$\sigma=\frac{M}{I} y$

$\mathrm{PA}=\mathrm{F}$
في الموقع الثاني و هذا يعزى الى الى اختلاف مكونات نسجة التربنتين أي اختلاف

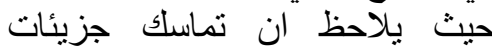

لاحتو ائها على نسبة كبيرة في الطين

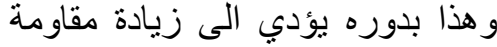

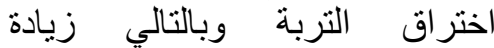
الاجهادات. [1] ويلاحظ زيادة الاجهادات بزيادة $x$

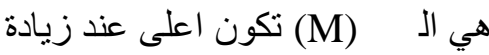

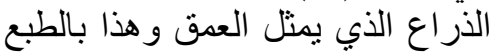

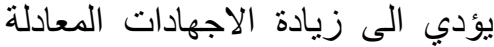
(2). [3] وهذا يعزى الى زئى زيادة الـادة والذي يزيد من قوة مقاومة (A) (P)

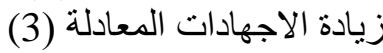
(4) يوضح مخطط اجهاد مع العمق .

في هذه الحالة الضغط و الذي يكون بمثابة الاجهاد على السطح. كما يمثل الثكل (5) توزيع القوى على مسـار السـاق تحت التربـة حيث ان اعلى قيمة عندة عند انف السـلاح عند اقصى عمق

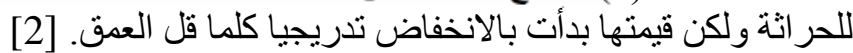



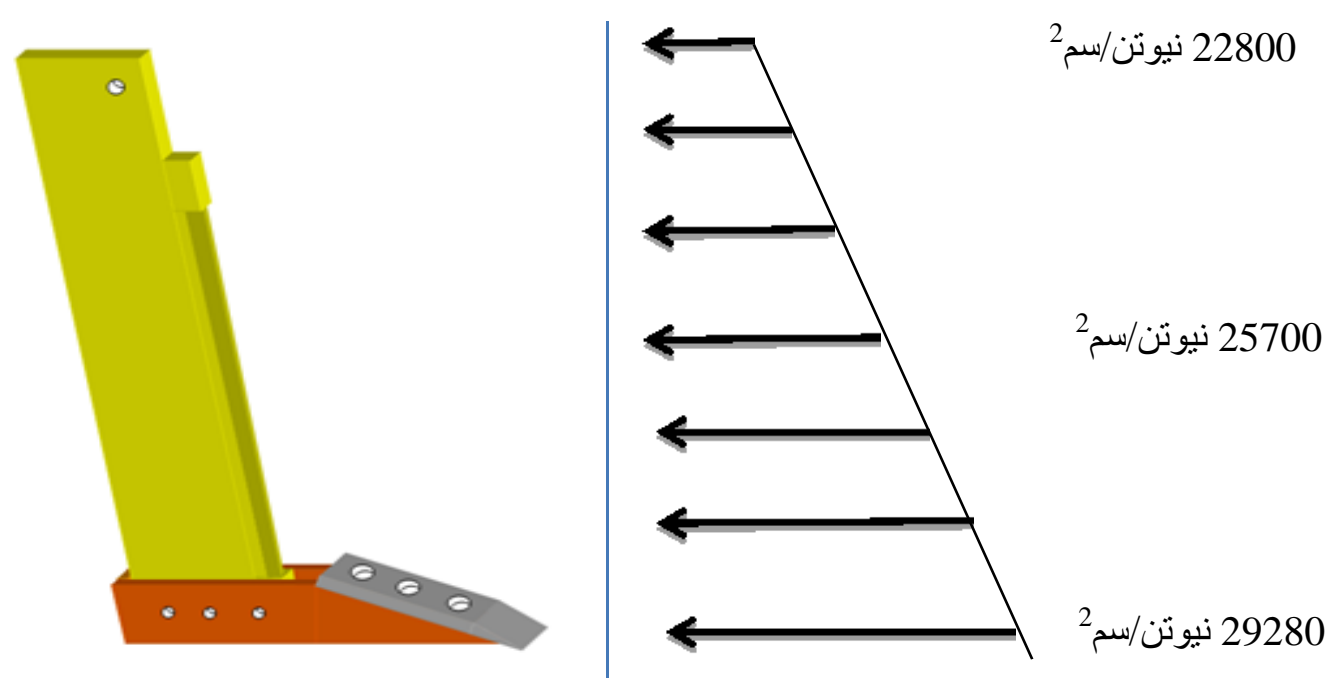

\section{(5) (5) توزيع القوى على}

الاستتناجات والتوصيات :

النتائج يمكن صيغة الاستوصيات : التنتاجات على النحو الاتي :

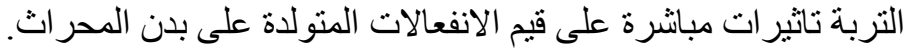

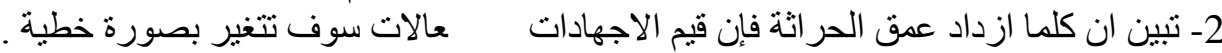

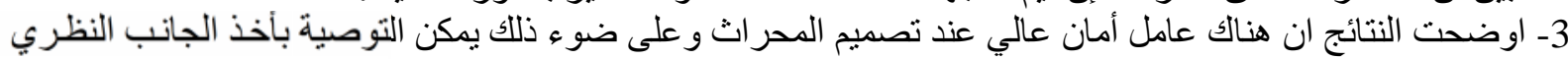

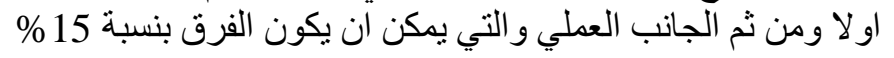

1. البنا، عزيز رمو ( ) 1990). معدات تهيئة التربة، جامعة الموصل، دار الكتب للطباعة و النشر، جمهورية العراق.

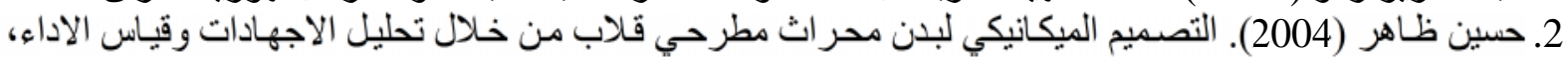

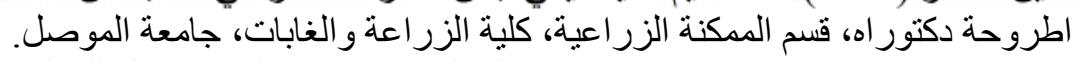

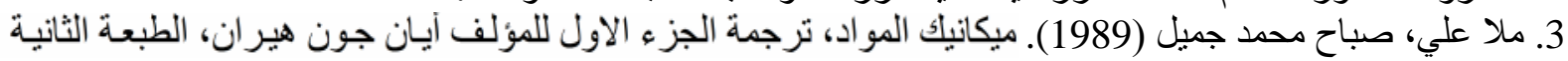

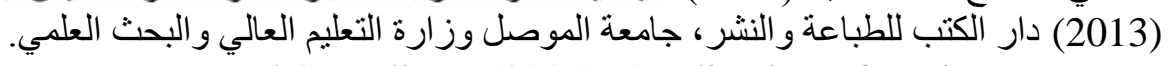

4. Adamchak, v.I; Morgan, M.T. and Sumali, H. (2001). Application of strain gauge array to estimate soil mechanical impendence on the 90. Tranactions of ASAE, 44(6); 1377-1383.

5. Agricultural Machinery Management Data. ASAE standards 2001 P.363

6. Hendry, A.W., 1968, Elements of Experimental stress Analysis. Printed in Great Britain by page Bros. (Norwich) L and d., Norwich.

7. Key to steel (DIN) 1998.

8. Mouazen A.M. and M. Nemenyi (1999). Finite element analysis of sub soiler cutting in non homogenous sandy loam soil. Soil and tillage Res.

9. Rohlf, R.Aand Wells, L.G. (2001). Determining matric stress with the modified com clay energy relationship. Transactions of ASAE, 44(5); 1047-1057.

10. Shackeford. J.F. "Failure Analysis" The Engineering Handbook Ed. Richard C. DorfBoca Raton; CRC Press LLC, 2000.

11. Wole Soboyejo, Marcel Dekker Mechanical Properties of engineered materials Inc. U.S.A. 2000.

$$
\text { تم اجراء البحث في كلية ألهندسة = جامعة ألموصل }
$$

